\title{
Theory of sunspot structure
}

\author{
John H.Thomas
}

Department of Mechanical Engineering and Department of Physics \& Astronomy, University of Rochester, Rochester, NY 14627, USA email: thomas@me.rochester.edu

\begin{abstract}
Recent high-resolution observations provide us with key information that allows us to begin to assemble a coherent theoretical picture of the formation and maintenance of a sunspot and its complex thermal and magnetic structure. A new picture of penumbral structure has emerged from observations, involving two components having different magnetic field inclinations and remaining essentially distinct over the lifetime of the spot, with little interchange of magnetic flux. The darker component, with more nearly horizontal magnetic field, includes "returning" magnetic flux tubes that dive down below the surface near the outer edge of the penumbra and carry much of the Evershed flow. The configuration of these flux tubes can be understood to be a consequence of downward pumping of magnetic flux by turbulent granular convection in the moat surrounding a sunspot. This process has been demonstrated in recent three-dimensional numerical simulations of fully compressible convection. The process of flux pumping is an important key to understanding the formation and maintenance of the penumbra, the hysteresis associated with the transition from a pore to a sunspot, and the behavior of moving magnetic features in the moat.
\end{abstract}

\section{Introduction}

Over the past decade we have seen substantial improvements in high-resolution observations of sunspots, leading to remarkable images with spatial resolution near $0.1^{\prime \prime}$ and spectra approaching this resolution (see the reviews by Solanki 2003 and Thomas \& Weiss 2004). These observations have revealed the complicated structure of sunspots in unprecedented detail, providing important clues to theoreticians seeking to understand sunspot structure on the basis of magnetohydrodynamic theory.

The most puzzling aspect of sunspot structure is the interlocking-comb geometry of the penumbral magnetic field. There is a systematic difference in the inclination (to the local vertical) of the magnetic field in the light and dark penumbral filaments, with the two components differing in inclination by $30^{\circ}-40^{\circ}$. At the umbra-penumbra boundary the fields in the bright and dark filaments are inclined at about $30^{\circ}$ and $60^{\circ}$, respectively, and the inclination in each component increases outward until the outer edge of the penumbra, where the field in the bright filaments is inclined at about $70^{\circ}$ and the field in the dark filaments is very nearly horizontal and in some cases inclined beyond $90^{\circ}$ in the form of "returning" flux tubes that dive back below the solar surface. This complicated configuration does resolve an apparent contradiction: on the one hand, much of a sunspot's magnetic flux must emerge through the penumbra, so the field must have a substantial vertical component (mostly in the bright filaments); on the other hand, the Evershed outflow (mostly confined to the dark filaments) is nearly horizontal and must be parallel to the magnetic field.

The magnetic field in the dark filaments either emerges from the sunspot at a shallow angle to form a low-lying magnetic canopy, or dives back down below the surface. The magnetic field in the bright filaments, however, typically emerges in the form of loops that extend over great distances on the Sun, as shown by X-ray observations (Sams, Golub \& Weiss 1992) and by EUV images from TRACE (Winebarger et al. 2002). In 
this configuration it is nearly impossible for there to be significant interchanges between the magnetic fields in the bright and dark filaments. Hence, the two components of the interlocking-comb magnetic field remain essentially distinct over the lifetime of the sunspot (Weiss et al. 2004).

\section{Axisymmetric models of pores and sunspots}

\subsection{Static models}

The simplest models of a pore or a sunspot ignore azimuthal variations and treat it as a meridional (poloidal) magnetic field confined to a flux tube of circular cross section, bounded by an azimuthal current sheet separating the tube from its nonmagnetic surroundings. Because the surrounding atmosphere is strongly stratified, the flux tube expands radially with height in order to maintain total pressure balance across the boundary. The radiative energy emerging from a sunspot is reduced by both the expanding cross-sectional area of the flux tube and magnetic inhibition of convection. This causes a greater superadiabatic temperature gradient in the tube, with the result that the sunspot appears cooler and darker and the visible surface of the umbra is lower than that of the surrounding photosphere (the Wilson depression).

Energy transport at the visible surface of the umbra is primarily radiative but must be primarily convective just below the surface. Models can be constructed by assuming a potential magnetic field and using the mixing-length theory of convective transport with a reduced mixing length inside the flux tube. The position of the azimuthal current sheet on the surface of the tube can then determined as a free boundary problem (Schmidt 1991). However, it is not possible to achieve complete pressure balance if the magnetic field within the tube is truly force free; instead, there must be azimuthal volume currents within the tube. This poses a difficult problem, which Jahn \& Schmidt (1994) showed can be simplified by concentrating all of these volume currents into a second, internal azimuthal current sheet representing the boundary between the umbra and penumbra. In their model the umbra is thermally insulated from the penumbra but some of the heat flux in the penumbra is supplied by heat transport from the hotter surroundings across the outer current sheet (the magnetopause). This approach leads to a family of models, of different total magnetic flux, that provide a reasonable description of the global (azimuthally averaged) structure of a sunspot.

\subsection{Dynamic models}

A static flux tube that fans out near the surface is concave toward its field-free surroundings and hence susceptible to a fluting interchange instability. The configuration is stabilized by the buoyancy force provided the radial component of the magnetic field at the surface of the tube decreases with height (Meyer, Schmidt \& Weiss 1977), which is generally true in the upper part of the flux tube. At some depth below the surface, however, where the buoyancy force is weak and the magnetic field is nearly vertical, the tube is unstable to interchanges. This instability would likely destroy the sunspot over a short time, so some dynamical effect must stabilize the tube. It is generally assumed that some sort of supergranule-scale "collar" flow in the surrounding gas accomplishes this stabilization.

One approach to studying this dynamical equilibrium is to simulate the formation of the sunspot flux tube by magnetoconvection in an idealized geometry. For example, Hurlburt \& Rucklidge (2000) modeled axisymmetric flux tubes confined by compressible convection in a cylindrical box. Their models show an increasing angle of inclination of the magnetic field at the edge of the tube with increasing total magnetic flux. In all cases 
the flux tube is surrounded by an annular convection cell with a downflow along the edge of the flux tube and a radial inflow near the surface. This agrees with observed flows for a pore but not for a fully developed sunspot, which has an outward surface flow in the surrounding moat. However, the models for larger total magnetic flux also have a counter-rotating outer annular cell, with surface flow directed radially outward. Hurlburt \& Rucklidge conjecture that for a sunspot the inner "collar" cell is hidden beneath the penumbra and only the outer "moat" cell is visible at the surface. This is an attractive picture, because it reconciles the expected presence of a downflow along a cooled sunspot flux tube and the observed outflow in the moat. More realistic simulations of the convective concentration of flux tubes are needed to check this conjecture.

\section{Formation and maintenance of the penumbra}

The magnetic field in an emerging active region is initially fragmented into many tiny flux elements which can accumulate between granules and mesogranules to form small pores. Some of these pores and flux elements then coalesce to form a larger pore and, eventually, a sunspot. As the total magnetic flux in a growing pore increases, the inclination of the field at the boundary of the pore increases and eventually reaches a critical value (about $35^{\circ}$ ), at which point the pore develops a penumbra. Observations show that the pore-sunspot transition is associated with hysteresis, in the sense that the largest pores are bigger than the smallest sunspots (Bray \& Loughhead 1964; Rucklidge, Schmidt \& Weiss 1995; Skumanich 1999).

We can imagine the growing pore to be an axisymmetric, expanding magnetic flux tube embedded in a stratified atmosphere. This flux tube is apparently stabilized against magnetic fluting even in the presence of convection, although convective motions inside and outside the pore must excite the stable fluting modes to some small amplitude (Tildesley \& Weiss 2004), thereby producing the narrow, finely fluted rim seen around even the tiniest pores (Scharmer et al. 2002).

A sequence of events in the transition between a pore and a fully developed sunspot has recently been proposed (Thomas et al. 2002; Weiss et al. 2004). The flux tube in a growing pore eventually becomes unstable to convectively driven filamentary perturbations, and the nonlinear development of this instability leads to mild fluting of the outer boundary and a rudimentary penumbra, as seen in protospots (Leka \& Skumanich 1998). This conjecture is supported by detailed calculations in cartesian geometry (Tildesley 2003; Tildesley \& Weiss 2004) and preliminary results in axisymmetric models (Hurlburt, Matthews \& Rucklidge 2000; Hurlburt \& Alexander 2002). Then there is a further jump to a fully developed penumbra with its interlocking-comb field configuration. (This rapid transition is observed to take place in twenty minutes or less: Leka \& Skumanich 1998; Yang et al. 2003.) The transition to a fully developed penumbra occurs when the depressed, more nearly horizontal spokes in the mildly fluted magnetic field are grabbed by sinking fluid in the surrounding granular convection and dragged downward by magnetic flux pumping. The flux pumping then keeps some of the magnetic field in the dark filaments submerged as the sunspot evolves further and decays, maintaining the penumbra even when the total magnetic flux remaining in the sunspot is less than that in the pore from which the sunspot formed. This provides a physical mechanism for the subcritical bifurcation proposed by Rucklidge, Schmidt \& Weiss (1995) to explain the hysteresis in the pore-sunspot transition.

The conjecture that magnetic flux pumping by granular convection is the key mechanism for the formation and maintenance of the penumbra is supported by numerical simulations, which are described in the next section. 


\section{Turbulent pumping of penumbral magnetic fields}

The concept of magnetic flux pumping arose from earlier concepts of flux expulsion, turbulent diamagnetism, and topological pumping, but the most important point is that in stratified compressible convection there is a strong contrast between the broad, gently rising plumes and the sinking fluid, which is focused into narrow, rapidly falling plumes. The magnetic field is governed by the induction equation and hence "feels" velocity rather than momentum density. Since the rising plumes expand, they transfer magnetic flux to the vigorous sinking plumes, which contract as they descend. Hence magnetic fields are pumped preferentially downwards. Such asymmetric turbulent pumping has been demonstrated in various numerical experiments concerned with transport of magnetic field from the convection zone into the stable layer beneath it, an important part of the solar dynamo process (Nordlund et al. 1992; Brandenburg et al. 1996; Tobias et al. 1998, 2001; Dorch \& Nordlund 2001; Ossendrijver et al. 2002).

Recent idealized numerical experiments have shown that flux pumping is also effective just below the solar surface because of the vigorous convection in the strongly superadiabatic granulation layer (Thomas et al. 2002; Weiss et al. 2004). Intense sinking plumes between granules and mesogranules in the moat drag magnetic flux downward in opposition to magnetic buoyancy and the weaker upflows. Below the granulation layer, pumping by the weaker supergranular convection is much less effective, allowing magnetic flux to accumulate at the base of the granulation layer. This provides a mechanism for submerging penumbral flux tubes in the moat surrounding a sunspot. We now describe these numerical experiments.

\subsection{The computational model}

The three-dimensional computational domain, representing a region just below the solar surface, consists of a rectangular box containing a fully compressible, electrically conducting, perfect gas (with $\gamma=5 / 3$ ), confined between two horizontal, impenetrable, stress-free boundaries. We assume that the shear viscosity, electrical conductivity, and gravitational acceleration are all constant. Within the domain there are two layers, an upper layer representing the surface granulation layer and a lower layer representing the upper convection zone. Each layer begins from a polytropic equilibrium state, and the polytropic indices $\left(m_{1}\right.$ in the upper layer, $m_{2}$ in the lower layer) and thermal conductivities are allowed to differ significantly between the two layers. The stability to convection of the initial polytropic state in each layer is determined by its polytropic index (for an adiabatically stratified layer $m_{\text {ad }}=3 / 2$ ). The upper layer is always chosen to be convectively unstable $\left(m_{1}=1.0\right)$. The relative stability of the two layers is measured by the stiffness parameter $S=\left(m_{2}-m_{\mathrm{ad}}\right) /\left(m_{\mathrm{ad}}-m_{1}\right)$. (An adiabatically stratified lower layer thus corresponds to $S=0$ ). In our initial calculations (Thomas et al. 2002), the stiffness parameter was $S=0.5$ and the lower layer was mildly subadiabatic. Subsequent calculations were performed with $S=0.0$ and -0.01 , corresponding to adiabatic $\left(m_{2}=1.5\right)$ and weakly superadiabatic $\left(m_{2}=1.495\right)$ lower layers (Weiss et al. 2004).

Periodic boundary conditions are imposed on all the fields in both horizontal directions. On the horizontal surfaces we impose stress-free, impermeable boundary conditions on the velocity field, constant temperature on the upper surface, and constant heat flux across the lower surface. The horizontal components of the magnetic field vanish on the upper and lower surfaces; this allow magnetic flux to escape from the domain by diffusion at the top or bottom. The basic equations of continuity, momentum, energy, and induction are integrated numerically as an initial value problem using a mixed finite-difference pseudo-spectral scheme. (Details of the numerical scheme can be found in Tobias et al. (2001). 


\subsection{Results of the simulations}

The flow is allowed to evolve in the absence of any magnetic field until a statistically steady convecting state has been established. Then we introduce a thin layer of uniform horizontal magnetic field $\left(B_{x}=0, B_{y}=B_{0}\right)$ in the middle of the upper layer, keeping total pressure balance between this layer and its surroundings by decreasing the density within the magnetized layer while the temperature remains unchanged. The decreased density in the magnetic layer causes motions driven by magnetic buoyancy, which combine with convectively driven motions to produce strands of locally strong magnetic field. Magnetic buoyancy and advection of magnetic flux by convective flows then compete in the subsequent evolution of the magnetic field.

Figures 1 shows results of a simulation (Weiss et al. 2004) with a mildly superadiabatic lower layer $(S=-0.01)$ and a very strong initial magnetic field strength (a nearly evacuated magnetic layer). Shown here are the patterns of vertical velocity and magnetic energy density at an early stage of the calculation, shortly after the magnetic layer was introduced, and a later stage, after significant pumping has occurred. Figure 1 also shows profiles of the average horizontal $(y)$ component of the magnetic field as a function of depth at various times for this calculation. Here one can see an initial rise of the magnetic field due to buoyancy and advection by upflows, followed by downward pumping of much of the remaining flux by the strong downflows, after which the maximum field lies just below the upper granulation layer.

Very recently we have carried out a simulation that better approximates the magnetic curvature forces that would arise because of the overall magnetic configuration of a sunspot. In this simulation (Brummell et al. 2004) we also add a thin slab of strong vertical magnetic field in the center of the box. This has the effect of strongly suppressing convective motions within the vertical slab, so that pumping of the horizontal field is inhibited there. As the horizontal field is pumped downward outside the vertical slab, the part of the field within the vertical slab lags behind and curvature forces are developed which impede the pumping. This mimics the situation in a penumbra, where the inner parts of penumbral flux tubes are held firmly within the overall sunspot flux bundle.

Figure 2 shows some preliminary results of this simulation, in the form of plots of randomly chosen magnetic field lines at different times. Although the pattern is complicated, one can identify field lines that have been pumped down to the base of the upper granular layer. Plots of the average horizontal field for this run look similar to those in Fig. 1 (for simulations without the vertical slab) and clearly show downward pumping.

Our idealized numerical experiments indicate that downward flux pumping of penumbral flux tubes is a robust effect. More realistic simulations are planned. A reasonable goal would be to produce a simulation that combines the production of a concentrated sunspot flux bundle by a large-scale (supergranular) convection cell and the downward pumping of penumbral flux by smaller-scale (granular) convection at the surface.

\section{Moving magnetic features in the moat}

The mechanism of magnetic flux pumping aids in our understanding of the moving magnetic features (MMFs) observed in the annular moat cell (with its persistent horizontal outflow at the surface) surrounding a mature sunspot. Small magnetic elements move radially outward across the moat with speeds ranging from a few tenths to $3 \mathrm{~km}$ $\mathrm{s}^{-1}$ (Sheeley 1969; Vrabec 1971; Harvey \& Harvey 1973; Zwaan 1992). Shine \& Title (2001) have classified these MMFs into three types, each of which can be interpreted in the context of the flux-pumping scenario. 

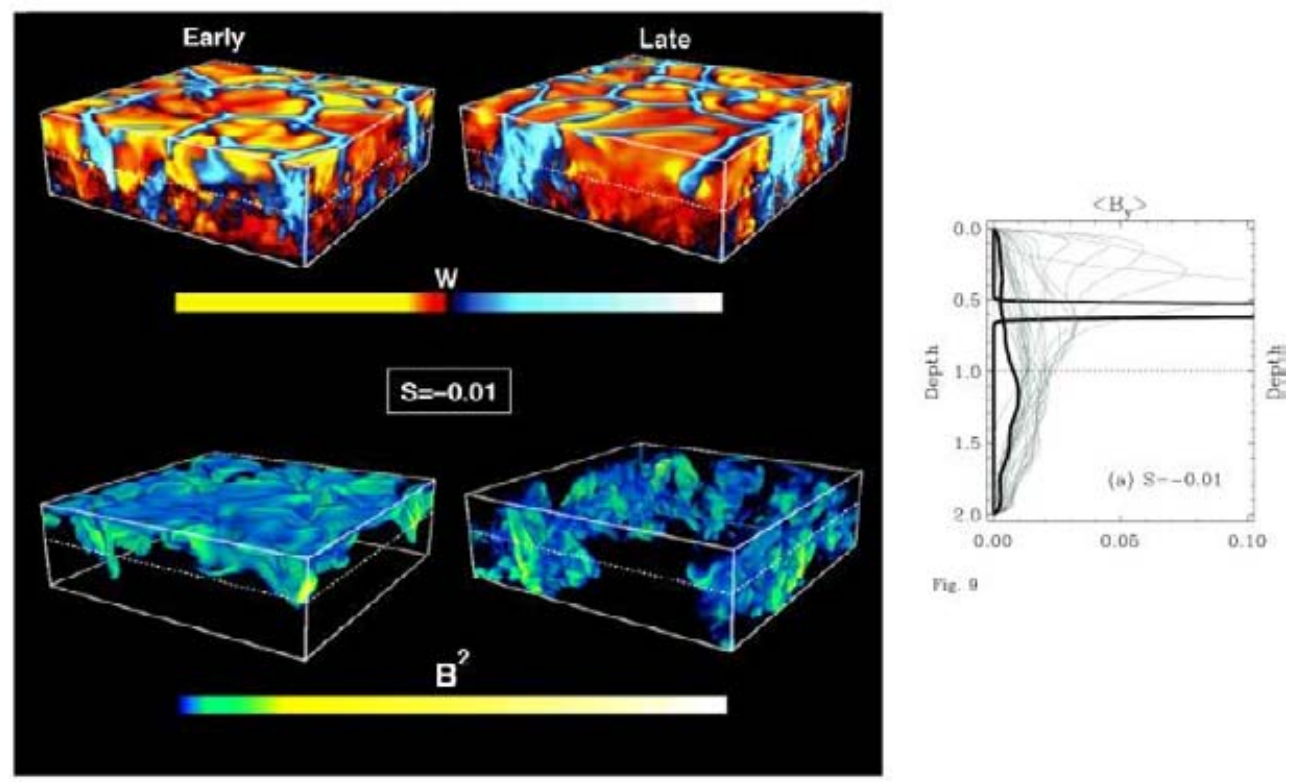

Fie. 9

Figure 1. Simulation of magnetic flux pumping by granular convection in the sunspot moat. Shown here are volume renderings of the vertical velocity $w$ and magnetic energy density $B^{2}$ at stages near the beginning and near the end of a calculation for stiffness parameter $S=-0.01$. (See text for further explanation.)

The type III MMFs are single magnetic elements, with magnetic polarity opposite to that of the sunspot, that move rapidly outward at speeds of 2 to $3 \mathrm{~km} \mathrm{~s}^{-1}$. These features can be produced when the pumping mechanism occasionally relaxes and allows a long section of a submerged flux tube to rise (through magnetic buoyancy and curvature forces) and emerge through the surface at a shallow angle, thus producing a rapid outward horizontal motion of the footpoint (the MMF) in the moat. Even while a penumbral flux tube remains submerged outside the sunspot, convective motions will cause it to bob up and down, producing rapid inward and outward excursions of the tips of dark penumbral filaments, as seen for example in the movies of Title et al. (1993).

Type II MMFs are single magnetic elements, with the same polarity as the sunspot, that move outward across the moat at speeds comparable to those of type I MMFs. These features are generally interpreted as flux tubes that have separated from the main flux bundle and are being carried away by the moat flow, thus producing the primary mechanism for the decay of a sunspot.

Type I MMFs consist of bipolar pairs of magnetic elements that move outward together across the moat at speeds of 0.5 to $1 \mathrm{~km} \mathrm{~s}^{-1}$. They usually first appear just outside the penumbra along a radial line extending from a dark filament. In the standard pattern (Harvey \& Harvey 1973; Shine \& Title 2001) the element of the pair nearest the sunspot has the same polarity as the sunspot. This pair can be interpreted as the footpoints of a 


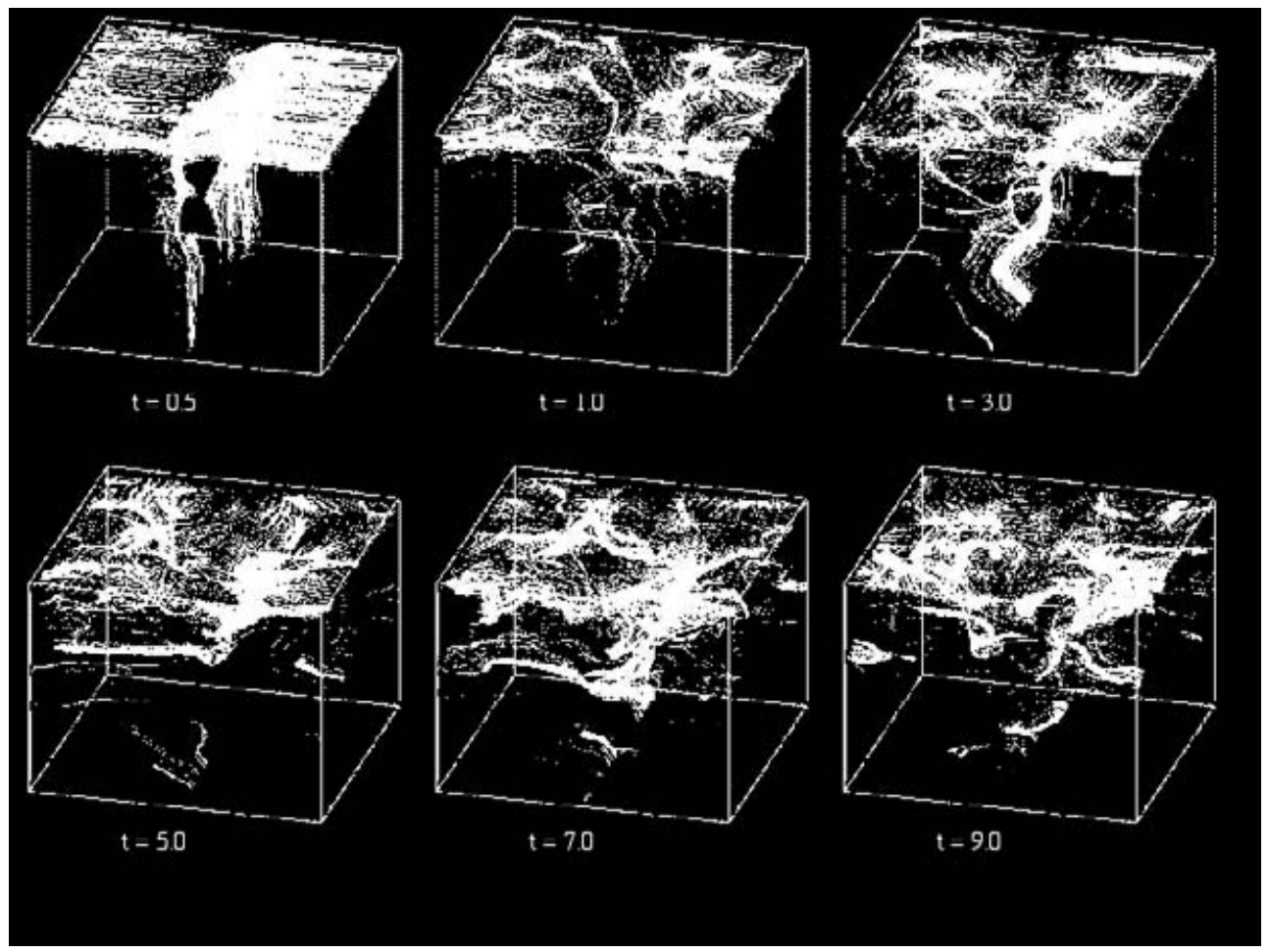

Figure 2. Plots of randomly chosen magnetic field lines at different times for a flux-pumping simulation similar to that shown in Figure 1, but with the addition of a vertical slab of strong magnetic field in the center of the box (see text).

magnetic loop that has erupted through the solar surface at a place along a submerged (downward-pumped) penumbral flux tube where there is a particularly strong convective updraft. The loop is then swept outward along with the granulation pattern by the moat flow. (The outward motion is often somewhat faster than the flow speed, however, possibly indicating that the motion is at least partly due to propagation as a solitary kink wave along the flux tube.) This picture is essentially the one proposed by Harvey \& Harvey (1973), but now flux pumping gives us a physical explanation for the general submergence of the flux tube and the formation of emerging loops along the tube. Several loops can emerge at different positions and at different times along the same flux tube; this is reflected in the fact that successive Type I MMFs tend to follow nearly identical paths across the moat. As long as a submerged flux tube remains attached to the sunspot, the Type I MMFs that form along it are not associated with the decay of the sunspot.

Two recent papers (Yurchyshyn, Wang \& Goode 2001; Zhang, Solanki \& Wang 2003), each based on observations of just two active regions, report a majority of type I MMF bipolar pairs with polarity arrangement opposite that of the "standard" arrangement described above, ie., with the feature farthest from the sunspot having the polarity of the spot. One active region (NOAA AR 8375), common to both these papers, was a young region showing a clear pattern of emergence of new magnetic bipoles, which should not be considered MMFs in the classical sense (V. Martínez Pillet, private communication). Perhaps some of the features reported in these papers were newly emerging bipoles 


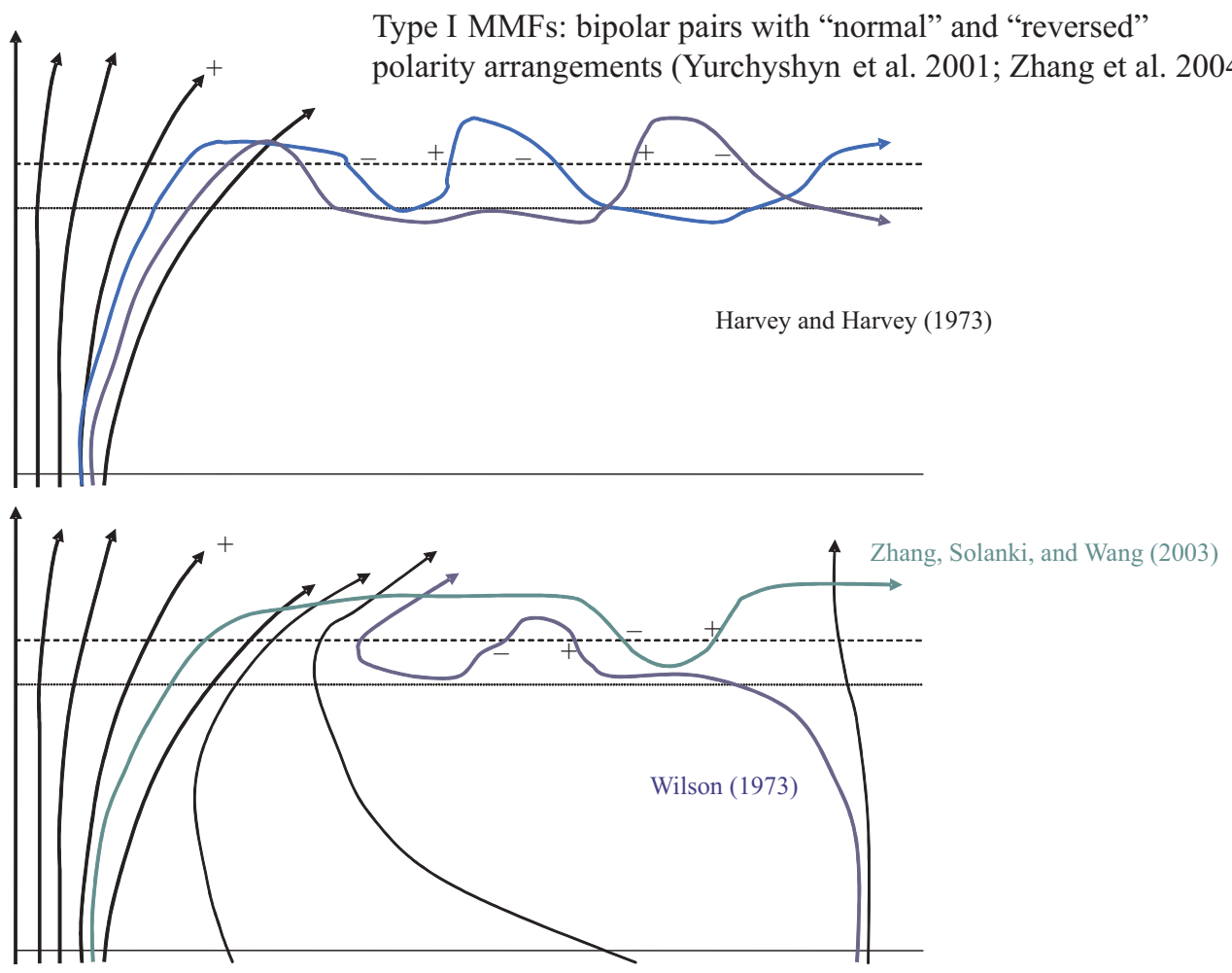

Figure 3. Various interpretations of type I bipolar moving magnetic features (MMFs).

unconnected with the sunspot. Granting, however, that some true type I MMFs have the reversed polarity arrangement, there are possible explanations. In the context of the Harvey \& Harvey (1973) picture, if there is more than one loop along the flux tube (the "sea-serpent" configuration shown in Fig. 3), then the assignment of features to pairs becomes somewhat ambiguous and depends on all footpoints along the flux tube being visible. Alternatively, the reversed polarity arrangement could be associated with the mechanism proposed by Wilson (1973), in which a flux tube is dragged away from the main flux bundle at a considerable depth, so that the field is in the opposite direction near the surface (see Fig. 3). Flux pumping could keep this flux tube submerged below the granulation layer, with occasional rising loops emerging with the reversed polarity arrangement. A different mechanism for the formation of the reversed-polarity type I MMFs has been proposed by Zhang et al. (2003). They suggest that these pairs form as a result of a downward kink that forms along the base of the canopy magnetic field, due to a dense packet of gas flowing outward as part of the Evershed flow (see Fig. 3). Even in this case flux pumping may play a role by submerging the kink once it is in contact with the granulation layer. 


\section{Summary}

Downward pumping of magnetic flux by turbulent convection in the surrounding granulation layer seems to be a key ingredient in explaining the formation and maintenance of the filamentary penumbra with its interlocking-comb magnetic field. This pumping mechanism provides a natural explanation for the submergence of the returning flux tubes in the moat, for the hysteresis associated with the pore-sunspot transition, and for the general behavior of the MMFs. The idealized numerical experiments we have carried out so far indicate that this flux pumping is a real and robust effect. More elaborate calculations, incorporating more realistic representations of the conditions in and around a sunspot, are needed to confirm this mechanism.

\section{Acknowledgements}

I thank Nigel Weiss, Steve Tobias, and Nic Brummell for their generous collaboration on the work on flux pumping, and Valentin Martínez Pillet for helpful discussions of MMFs.

\section{References}

Brandenburg, A., Jennings, R. L., Nordlund, Å., Rieutord, M., Stein, R. F. \& Tuominen, I. 1996. J. Fluid Mech. 306, 325.

Bray, R. J. \& Loughhead, R. E. 1964. Sunspots (London: Chapman and Hall).

Brummell, N. H., Tobias, S. M., Thomas, J. H., and Weiss, N. O. 2004. (In preparation).

Dorch, S. B. F. \& Nordlund, Å. 2001. Astron. Astrophys. 365, 562-570.

Harvey, K. \& Harvey, J. 1973. Sol. Phys. 28, 61-71.

Hurlburt, N. \& Alexander, D. 2002. In COSPAR Colloquia Ser. 14: Solar-Terrestrial Magnetic Activity and Space Environment, ed. H Wang \& R Xu )Oxford, UK: Pergamon Press), pp. 19-25.

Hurlburt, N. E., Matthews, P. C. \& Rucklidge, A. M. 2000. Sol. Phys. 192, 109-118.

Hurlburt, N. E. \& Rucklidge, A. M. 2000. Mon. Not. Roy. Astron. Soc. 314, 793-806.

Jahn, K. \& Schmidt, H. U. 1994. Astron. Astrophys. 290, 295-317.

Leka, K. D. \& Skumanich, A. 1998. Astrophys. J. 507, 454-469.

Meyer, F., Schmidt, H. U. \& Weiss, N. O. 1977. Mon. Not. Roy. Astron. Soc. 179, 741-761.

Nordlund, Å., Brandenburg, A., Jennings, R. L., Rieutord, M., Ruokalainen, J., Stein, R. F. \& Tuominen, I. 1992. Astrophys. J. 392, 647-652.

Ossendrijver, M., Stix, M., Brandenburg, A. \& Rüdiger, G. 2002. Astron. Astrophys. 394, 735-

Rucklidge, A. M., Schmidt, H. U. \& Weiss, N. O. 1995. Mon. Not. Roy. Astron. Soc. 273, 491-498.

Sams, B. J., III, Golub, L. \& Weiss, N. O. 1992. Astrophys. J. 399, 313-317.

Scharmer, G. B., Gudiksen, B. V., Kiselman, D., Löfdahl, M. G. \& Rouppe van der Voort, L. H. M. 2002. Nature 420, 151-153.

Sheeley, N. R. Jr. 1969. Sol. Phys. 9, 347-357.

Shine, R. A. \& Title, A. M. 2001. In Encyclopedia of Astronomy and Astrophysics (Nature Publishing Group), p. 3209.

Skumanich, A. 1999. Astrophys. J. 512, 975-984.

Solanki, S. K. 2003. Astron. Astrophys. Rev. 11, 153-286.

Thomas, J. H. \& Weiss, N. O. 1992. In Sunspots: Theory and Observations, ed. J. H. Thomas \& N. O. Weiss (Dordrecht: Kluwer), pp. 3-59.

Thomas, J. H. \& Weiss, N. O. 2004. Ann. Rev. Astron. Astrophys. 42, 517-548.

Thomas, J. H., Weiss, N. O., Tobias, S. M. \& Brummell, N. H. 2002. Nature 420, 390-393. 
Tildesley, M. J. 2002. Mon. Not. Roy. Astron. Soc. 338, 497-507.

Tildesley, M. J. \& Weiss, N. O. 2004. Mon. Not. Roy. Astron. Soc. 350, 657-670.

Title, A. M., Frank, Z. A., Shine, R. A., Tarbell, T. D., Topka, K. P., Scharmer, G. \& Schmidt, W. 1993. Astrophys. J. 403, 780-796.

Tobias, S. M., Brummell, N. H., Clune, T. L. \& Toomre, J. 1998. Astrophys. J. 502, L177-L180.

Tobias, S. M., Brummell, N. H., Clune, T. L. \& Toomre, J. 2001. Astrophys. J. 549, 1183-1203.

Vrabec, D. 1971. In IAU Symposium 43, Solar Magnetic Fields, ed. R. Howard (Dordrecht: Reidel), pp. 329-339.

Weiss, N. O, Thomas, J. H., Brummell, N. H. \& Tobias, S. M. 2004. Astrophys. J. 600, 10731090.

Wilson, P. R. 1973. Sol. Phys. 32, 435-439.

Winebarger, A. R., Warren, H., van Ballegooijen, A., DeLuca, E. E. \& Golub, L. 2002. Astrophys. J. 567, L89-L92.

Yang, G., Xu, Y., Wang, H. \& Denker, C. 2003. Astrophys. J. 597, 1190-1199.

Yurchyshyn, V. B., Wang, H. \& Goode, P. R. 2001. Astrophys. J. 550, 470-474.

Zhang, J., Solanki, S. K. \& Wang, J. 2003. Astron. Astrophys. 399, 755-761.

Zwaan, C. 1992. In Sunspots: Theory and Observations, ed. J. H. Thomas and N. O. Weiss (Dordrecht: Kluwer), pp. 75-100. 